\title{
Traumatic Brain Injury Rehabilitation in Hong Kong: A Review of Practice and Research
}

\author{
Junhong Yu, ${ }^{1,2}$ Helena M. K. Tam, ${ }^{1,2}$ and Tatia M. C. Lee ${ }^{1,2,3}$ \\ ${ }^{1}$ Laboratory of Neuropsychology, The University of Hong Kong, Pokfulam, Hong Kong \\ ${ }^{2}$ Institute of Clinical Neuropsychology, The University of Hong Kong, Pokfulam, Hong Kong \\ ${ }^{3}$ The State Key Laboratory of Brain and Cognitive Sciences, The University of Hong Kong, Pokfulam, Hong Kong \\ Correspondence should be addressed to Tatia M. C. Lee; tmclee@hku.hk
}

Received 26 May 2015; Revised 21 August 2015; Accepted 3 September 2015

Academic Editor: Solrun Sigurdardottir

Copyright (C) 2015 Junhong Yu et al. This is an open access article distributed under the Creative Commons Attribution License, which permits unrestricted use, distribution, and reproduction in any medium, provided the original work is properly cited.

Background. The rising public health concern regarding traumatic brain injury (TBI) implies a growing need for rehabilitation services for patients surviving TBI. Methods. To this end, this paper reviews the practices and research on TBI rehabilitation in Hong Kong so as to inform future developments in this area. This paper begins by introducing the general situation of TBI patients in Hong Kong and the need for rehabilitation. Next, the trauma system in Hong Kong is introduced. Following that is a detailed description of the rehabilitation services for TBI patients in Hong Kong, as exemplified by a rehabilitation hospital in Hong Kong. This paper will also review intervention studies on rehabilitating brain-injured populations in Hong Kong with respect to various rehabilitation goals. Lastly, the implications of culture-related issues will be discussed in relation to TBI. Results/Conclusions. The intervention studies conducted in Hong Kong are generally successful in achieving various rehabilitative outcomes. Additionally, certain cultural-related issues, such as the stigma associated with TBI, may impede the rehabilitative process and lead to various psychosocial problems.

\section{Introduction}

Traumatic brain injury (TBI) is a condition involving brain damage caused by external forces, such as acceleration and deceleration, impact, blast waves, or penetrating injury. Its pathophysiology is characterized by shearing of white matter tracts, intra- and extracerebral hematomas, focal contusions, and diffuse swelling [1]. This condition has affected many people around the world; every year at least 42 million people sustain a TBI [2]. These survivors of TBI are likely to suffer from many possible long-term consequences in emotional, cognitive, and daily functioning [3-5].

Similar to findings from studies conducted in Western populations [6], survivors of TBI in Hong Kong also report similar consequences following their brain injury such as having a low quality of life. Specifically, they are unsatisfied with their material well-being, their place in the community, and their productivity at work [7]. This dissatisfaction may very likely be due to their reduced ability to work to support themselves and thereby contribute to the community. In fact, the postinjury employment rate of brain-injured individuals in Hong Kong ranges from $10 \%$ to $47 \%[7,8]$, which is relatively low compared to their Western counterparts [9]. Another possible reason for their low quality of life may revolve around interpersonal issues commonly affecting survivors of TBI. Those who had been living with brain injuries for an extended period of time ( $\geq 5$ years) reported low satisfaction in the domain of intimacy [7], suggesting that they have experienced some strained relationships with others. The aging population in Hong Kong [10] further complicates the local TBI context in two ways. First, the elderly population is highly prone to falls [11] and falls are the most common cause of trauma-related injuries within the Hong Kong context [12]. Hence, as the population grows, we would expect the incidence of TBI to increase correspondingly. Second, relative to younger brain-injured patients, elderly patients require longer hospitalizations and have poorer functional outcomes [13, 14].

According to Yeung et al. [15], in Hong Kong approximately a fifth of all emergency cases received by hospitals 
were trauma related, and more than half of these cases were diagnosed with TBI [12]. The increasing incidence of TBI is paired with a growing need and importance for rehabilitative services catered to those afflicted with TBI. TBI patients, even those who suffer from mild but repetitive TBI, may suffer long-term consequences, such as progressive brain atrophy and increased vulnerability to neurodegenerative disorders [16]. Within the cognitive domain, those with moderate to severe TBI are likely to be impaired in attention, memory, executive functions, and insight relating to their deficits [4, 17]; these impairments will in turn have an adverse impact on functional outcomes [18]. Those with mild TBI are not spared from cognitive impairments either; they may still report clusters of attentional impairments and deficits in verbal fluency [19] despite sustaining the injury years ago [20]. They also experience difficulties in the psychosocial domain-often as a result of a combination of injury-related, psychological, and social factors $[21,22]$, which predisposes them to a range of psychosocial and emotional problems [5]. Taken together, these consequences also hinder their reintegration into the community [23]. Fortunately, some of these consequences are at least amenable to rehabilitation. In a review of randomized controlled trials (RCTs) targeting various rehabilitation goals in patients with moderate to severe TBI, 36 out of 45 RCTs reported significant positive gains in the cognitive, functional, and/or psychosocial domains [24]. The need for rehabilitative efforts to better the lives of TBI patients cannot be understated; rehabilitation for TBI is a key area to direct research and intervention efforts so as to better patients' outcomes in Hong Kong. While there has been extensive work in the literature on the rehabilitation of TBI patients, most of this research has been conducted with Western populations and in managed care settings; research in the Asian contexts is lacking. Moreover, Asian cultural factors in TBI rehabilitation have often been overlooked in the literature. Given that health care infrastructure and culture are major factors in shaping rehabilitative efforts $[25,26]$, there might be issues with translating these findings into practice in Hong Kong - a society with a predominantly Chinese population where healthcare services are typically financed by government subsidies and out-of-pocket payments (by the patients and/or their families), instead of relying on social or private insurance. Hence, whenever possible, it may be more useful to look at the practice and research in the local context, so as to inform local TBI rehabilitation efforts. To this end, the current report aims to review the practice and research of rehabilitating TBI patients in Hong Kong as well as explore the implications of TBI in the local cultural context. The present review is focused only on the rehabilitation of the adult TBI population (i.e., aged 18 years and above) in Hong Kong.

\section{Description of TBI Practices/Health Care Services/Rehabilitation in Hong Kong}

2.1. Trauma System. Patients who sustain trauma-related injuries are served by the trauma system as managed by the Hospital Authority (HA). The current trauma system, comprising five regional trauma centers-namely, Prince of Wales Hospital, Princess Margaret Hospital, Queen Elizabeth Hospital, Queen Mary Hospital, and Tuen Mun Hospitalwas set up gradually in 2003 to provide trauma care services to various hospital clusters. For instance, Queen Elizabeth Hospital, which is based in central Kowloon, serves one such cluster by extending its trauma services to three other hospitals within its close proximity [27]. These five trauma centers had met the criteria for a Level I trauma center and were well equipped to handle trauma cases [28]; a Level I trauma center has to be able to admit at least 1,200 trauma patients or receive 240 admissions of severe injuries in a year [28]. In the past, trauma casualties were brought to the nearest public hospital regardless of the severity of their injuries. With the pilot implementation of the Primary Trauma Diversion Policy in 2003, trauma casualties are transported from the scene to these trauma centers directly if necessary, as determined by the ambulance crew according to a standardized protocol [29]. Alternatively, the crew may decide to transport the casualty to a regional hospital for initial resuscitation before transferring to a trauma center, that is, a Secondary Trauma Diversion [30]. Prehospital trauma care is provided by the ambulance services; the paramedics were trained and equipped to provide such care on the way to the hospital [31]. Subsequently, a trauma team consisting of an emergency physician, trauma surgeon, and other specialists [32] is called upon to attend to the case received at the trauma center [33]. These trauma centers provide a variety of services for the acute care and rehabilitation of TBI patients. On top of that, there are a few convalescent hospitals that specifically cater to their rehabilitative needs, such as Tung Wah Eastern Hospital, MacLehose Medical Rehabilitation Centre, Rehabid Centre, Rotary Rehabid Centre for Children, and The Duchess of Kent Children's Hospital. These rehabilitation units provide a range of services for neurological patients in general. However, due to limited resources, some patients with TBI may also receive rehabilitation services from other hospitals. Figure 1 depicts the flow of trauma case management.

2.2. Rehabilitation. For the purpose of describing the rehabilitation of TBI patients in Hong Kong, we shall examine a rehabilitation hospital in Hong Kong: the MacLehose Medical Rehabilitation Centre (MMRC) [34]. This example was selected due to its significance of being the first and only rehabilitation hospital opened on Hong Kong Island. In this hospital, rehabilitation regimes are tailored according to each patient's assessment findings and are delivered by a multidisciplinary team. For TBI cases, this team would usually include clinical psychologists, occupational therapists, physiotherapists, and speech therapists, in addition to other medical professionals. Cognitive rehabilitation is conducted by the clinical psychologists. Based on the concept of neuroplasticity, well-structured cognitive training programs, targeting attention, memory, visual-spatial, and executive functions, are delivered to TBI patients. The occupational therapists assist the patients to work on their self-care skills and household management and modification, as well as 


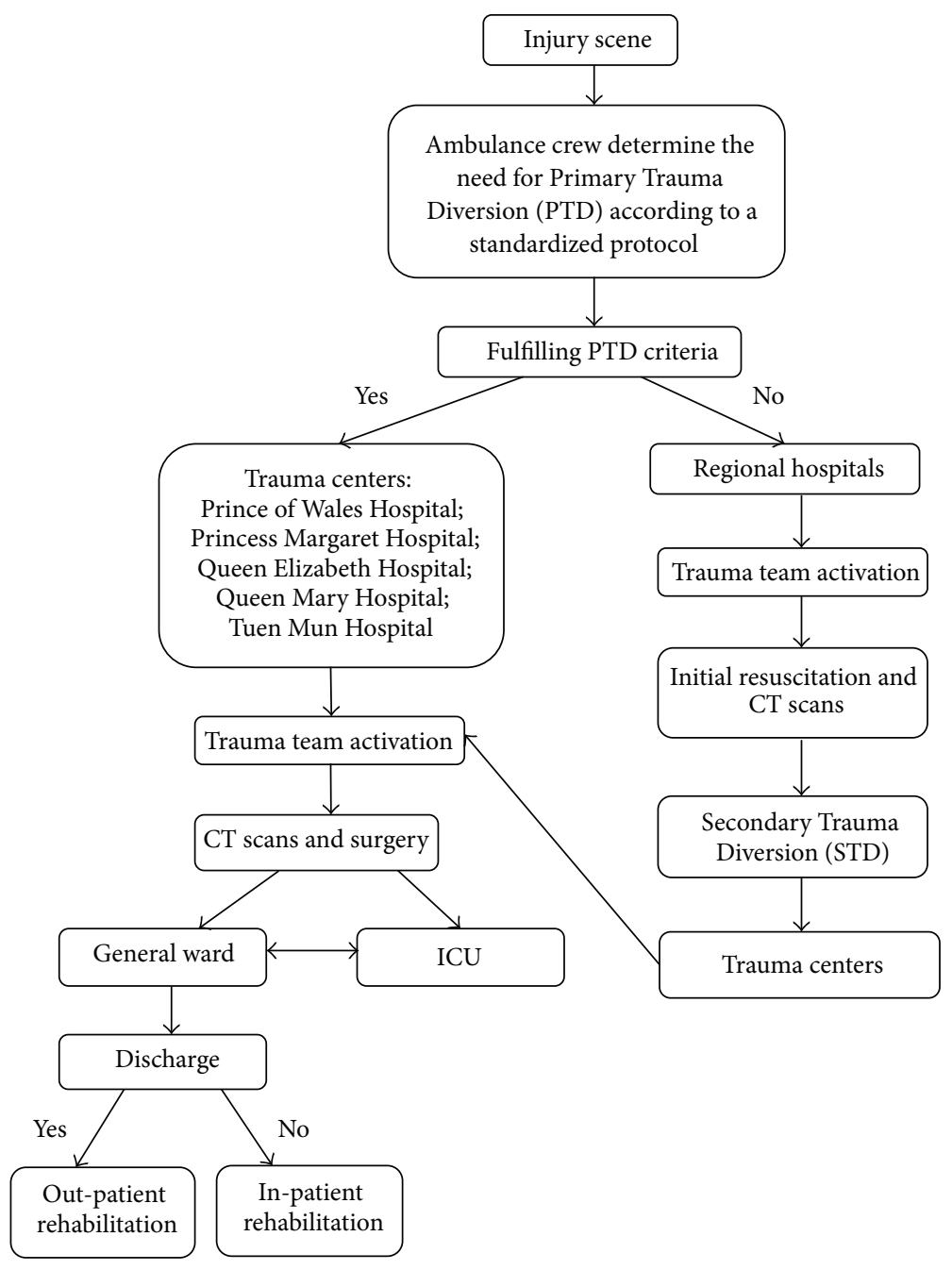

FIGURE 1: Flow diagram illustrating trauma patient management.

community integration. The physiotherapy team implements a rehabilitation program to maximize the patients' existing physical abilities and to regain functional independence. For this purpose, they are equipped with a neurological room, a standard sized gymnasium, and a hydrotherapy room. The speech therapists attend to patients who have issues with swallowing and/or verbal communication. These different specialists work closely with each other to contribute to the rehabilitation process and to carry out interventions tailored to each patient. They also meet up in weekly case rounds to discuss the progress of each patient. After a patient has completed his/her assigned rehabilitation regime, predischarge arrangements are made by the same team.

Patients usually receive short-term rehabilitation services in hospital settings. They may however, with an appropriate referral, turn to various nongovernmental organizations (NGOs) in Hong Kong for long-term care and rehabilitation. These NGO rehabilitation centers provide various outpatient services for discharged neurological patients and also facilitate the patient's reintegration into the community.

\section{Review of TBI Rehabilitation Research in Hong Kong}

At the time of this writing, there have not been any published trials on the rehabilitation of, specifically, TBI patients in Hong Kong. Given the paucity of TBI research in Hong Kong, it might be worthwhile to broaden the scope to other brain-injured populations, such as that of stroke. Stroke and TBI are highly similar in terms of pathophysiology. Hence, rehabilitation strategies beneficial to patients with stroke are likely to be beneficial to those with TBI as well [35]. For the present review, literature search was conducted using PubMed and Scopus with the following search terms in the title or abstract of the article: traumatic brain injury, TBI, close head injury, stroke, intervention, RCT, trial, training, remediation, rehabilitation, and Hong Kong. The exclusion criteria were (1) nonintervention studies, (2) studies that were not conducted in the postacute phase of the brain injury, (3) studies that did not include brain-injured participants, (4) studies that included participants below the age of 18 years, (5) studies that were conducted outside Hong Kong, and (6) 
studies that did not focus on functional related outcomes or had focused on narrow areas of physical functioning (i.e., hemiparetic upper extremity). A total of 7 studies were retrieved with the above criteria.

These studies have achieved various significant rehabilitative outcomes among brain-injured patients in Hong Kong via different modalities and objectives. For instance, improving the daily functioning of these patients is one such objective; these patients are likely to be impaired in many aspects of their daily functioning [36, 37]. To remediate such impairments, two interventions utilized relearning protocols to enhance competency on tasks with which patients had difficulty with following stroke. In the first, the intervention utilized mental imagery to achieve patients' goals [38]. Patients $(n=49)$ were randomly assigned to the mental imagery group or functional retraining group for three weeks $(5 \mathrm{~h} / \mathrm{wk})$. In the mental imagery group, patients were taught to use mental imagery to analyze and identify their difficulties and practice certain tasks. The functional retraining group practiced similar tasks; however, unlike the mental imagery group, the entire process was more instructional and didactic and did not involve mental imagery. At the end of the intervention, the mental imagery group had significantly higher levels of task performance on both trained and untrained tasks.

The other relearning intervention focused on the relearning of motor skills [39]. A total of 66 patients were randomly assigned to a motor-relearning program or conventional therapy program for six weeks $(6 \mathrm{~h} / \mathrm{wk})$. The motorrelearning program involved a step-by-step process of first identifying one's motor task-related deficit, remediating this deficit by practicing on selected tasks related to the deficit, and transferring the skills acquired to functional tasks. The conventional therapy program was similar, except that the task selection was based on the patients' physical status rather than their deficits and patients were not trained to identify their deficits. At the end of six weeks, group $\times$ time interaction effects suggested that the motor-relearning program had produced better outcomes relative to the conventional therapy program on measures of functional balance, self-care ability, daily living tasks, and community integration.

Stroke and TBI patients may both experience problems related to mobility [3, 40]; hence, it is important for community-based interventions to reach out to these people, who may have difficulties accessing rehabilitative facilities far away from home. There were three studies that specifically tackled such issues. The first study [41] made use of home visits within a nurse-led transitional care RCT. Stroke patients $(N=108)$ were randomly distributed between the intervention and control groups. The intervention group was administered a holistic care package that was delivered via weekly home visits that involved motivational interviewing and follow-up telephone calls for four weeks. Subsequently, the intervention group reported better physical functioning relative to controls immediately after the intervention and at a follow-up assessment four weeks later.

The second study [42] had video conferencing apparatus sited at various community venues in a district to reach out to their participants. Stroke patients $(N=21)$ took part in an eight-week intervention $(1.5 \mathrm{~h} / \mathrm{wk})$ that consisted of stroke education and physical exercise components, both of which were delivered via video conferencing to all participants. At the end of eight weeks, there were significant increases, relative to baseline, in measures of physical balance ability, self-esteem, quality of life, and knowledge of stroke. It should be noted that these results should be interpreted conservatively since there were no comparisons to a control group.

The third study [43] examined the option of having a short-term residential care program to eliminate the need for patients to travel. In this study, 188 stroke patients were assigned either to the residential care program or to a usual care program (as similarly described in the previous section) in a public hospital (both $12 \mathrm{~h} / \mathrm{wk}$ ). The residential care program had similar rehabilitation provisions as the usual care program, though their rehabilitation regime was spread out across more days of the week. Additionally, in the former, participants stayed in a home-like environment instead of hospitals and received round-the-clock residential and nursing care. At the end of four months, both groups had similar levels of improvements in general cognition, daily functioning, caregiver burden, depression, and self-esteem.

Brain injuries can also result in a variety of cognitive deficits as mentioned earlier. Memory impairments are one of these deficits that was of key interest in Tam and Man's [44] intervention study. In their study, 34 brain-injured patients with impairments in semantic memory were assigned to four different computerized training groups for two weeks $(2.5 \mathrm{~h} / \mathrm{wk})$ and a no-treatment control group. Those in the training groups were trained to remember similar contents across groups, such as faces and names, things to do, something that was said, and where to place an item. However, the training strategy differed across groups: The first group was allowed to work at their own pace; the second group was given clear, consistent, and nonjudgmental feedback at every instance; the third group made use of actual stimuli that the participants had seen or known before; and the last group utilized attractive and bright stimuli. Even though all four training groups showed significant performance improvements across time on the computerized tasks within their training programs, none of the training groups reported significant improvements across time on a standardized memory assessment (i.e., Rivermead Behavioural Memory Test).

Attention is another cognitive domain where intervention work could be targeted. In one such experiment [45], 10 patients with closed head injury (CHI) were compared with 10 healthy controls on jigsaw puzzle tasks. All participants completed two different jigsaw puzzles while being recorded on video. In one of the puzzle tasks, they were instructed to verbalize their actions, and in the other no such instructions were given. Also, in the course of completing a puzzle, distractors (such as someone dropping a book or playing on the computer) were introduced. The authors divided the recorded videos into fifteen-second clips in which they coded for the presence of a distractor and the presence of offtask behaviors (e.g., head and eyes oriented away from the puzzle). The numbers of correct and incorrect placements of 
the puzzle pieces were also recorded. The results indicated that verbalization did have a significant effect on reducing the number of off-task behaviors in the presence of distractors in both $\mathrm{CHI}$ patients and controls. It was also reported that there were significantly more correct placements in the verbalization condition than the nonverbalization condition in both groups. In both outcome measures, the differences attributed to verbalization were larger for $\mathrm{CHI}$ patients than for controls. The authors, while noting possible ceiling effects on the controls, suggested that CHI patients, relative to controls, can benefit more from such verbalization techniques.

The results of these intervention studies in Hong Kong are generally consistent with previous research in showing that functional outcomes among the brain-injured, such as those within the physical, psychosocial, and cognitive domains, can be improved via various rehabilitative approaches [24]. However it should also be noted that most of these studies do not meet Cicerone et al.s [46] criteria of a well-designed "Class I" study for their findings to be translated into practice standards and guidelines.

\section{Cultural Implications of TBI in the Local Hong Kong Context}

The disabilities or dysfunctions associated with TBI may not be the only concerns of TBI patients. TBI patients may experience certain psychosocial problems, which have less to do with the severity of the injury but perhaps more so to do with societal perceptions [47]. These societal perceptions or stigma may also have major implications for TBI patients' rehabilitation. The discussion of stigma has a special relevance here in Hong Kong, given the cultural context and the nature of the public health system.

One cannot easily identify a TBI patient just from his/her appearance because TBIs are usually not associated with any physically obvious abnormalities unlike patients with physical disabilities. Hence, individuals with TBI can choose whether to conceal their disabilities or disclose them to others. In either scenario, there will be undesirable consequences. If they disclose their condition to others, they might risk being stigmatized by the society as being mentally ill [47]. This is due to a number of reasons: (1) similar to those afflicted with mental illness, TBI patients do sometimes exhibit deviant behaviors and mental disabilities/impairments as well [48], and as a result, the layperson may not be able to distinguish between neurological conditions and mental illnesses [49]; (2) TBI patients are often diagnosed with mental illness following TBI [50]; and (3) the label of "braininjured," like "mentally ill," is equally reductionistic and carries similar negative connotations for the individual [47]. Furthermore, this stigma can be particularly unpleasant in Hong Kong because of the traditional Chinese belief that mental illness reflects the inferiority of one's family, the failure of one's parents, and the misdoings of one's ancestors [51]. In essence, having a mentally ill relative is something to be ashamed of in the Chinese context. This stigma or shame adds further stress to the family, who are already burdened by the need to provide and care for their brain-injured relatives.
Within Chinese contexts, families assumed the primary role in the management, coordination, and provision of care for TBI patients. However, these families were generally not adequately equipped in terms of resources and information to deal with the challenges of caring for their brain-injured relatives. As a result of undertaking these responsibilities, most of these families are already overwhelmed with shock, negative emotions, and uncertainty and consequently compromised their own physical and psychological well-being in the process $[52,53]$.

As a result, it is likely that TBI patients, like those diagnosed with mental illness, would choose to conceal their conditions from others to avoid the stigma or even hide their condition from their family members to avoid implicating them [54]. In fact, this tendency to cover up has been documented among TBI patients from a similar cultural background in a qualitative study. In this study, Vietnamese respondents reported attempting to cover up or not draw attention to their TBI by avoiding friends and telling lies because they were worried it might bring shame to their families [26]. This culture of concealing one's condition makes it difficult for people with TBI to seek social support, access rehabilitative services, and receive social welfare benefits. In Hong Kong, TBI is considered as a form of disability and TBI patients-like those who are physically disabled-may apply to receive social welfare assistance to cope with their disability. However, in order to obtain these benefits, one has to be certified "severely disabled" by the authorities [55], and this label may deter TBI patients from applying for these benefits especially if they would prefer to not draw attention to their conditions or disabilities. Furthermore, by concealing their disabilities, TBI patients receive less sympathy and empathetic understanding for their inappropriate actions and deficits, and as a result, unrealistic expectations are often placed upon them at the workplace based on the failure to compensate for their disabilities [56]. Even among family members who are well aware of the patient's condition, their lack of understanding of the residual impairments of TBI will similarly result in such unrealistic expectations. For instance, family members and caregivers would assume a hospital discharge implies that the patient will be able to revert back to his normal life, such as by returning to work, especially since the patient does not present with any obvious physical abnormalities that might indicate functional impairments. These unrealistic expectations set upon individuals with TBI will further complicate their interpersonal relationships and occupational functioning. The failure to satisfy such expectations will adversely affect the patient's self-concept as well. This is especially so in the Chinese culture whereby having a job symbolizes good health [8], and work itself is a major aspect of one's self-concept [57].

Taken together, the stigma encountered by TBI patients can hinder their rehabilitation in more than one way. This is a major concern that has yet to be adequately addressed in the current practices and research on rehabilitating TBI patients in Hong Kong. Certainly, more effort should be devoted to tackling such stigma. One effective way in which this can be accomplished is to promote more interpersonal contact with and exposure to this group of people. This method was 
found to be more effective than education alone in reducing various aspects of the stigma and can be implemented widely via school-based programs [58].

\section{Future Directions}

The current rehabilitation practices for TBI patients in Hong Kong have generally satisfied the guidelines for the rehabilitation following acquired brain injury as laid out by the Royal College of Physicians and British Society of Rehabilitation Medicine [59]. TBI patients in Hong Kong were also generally satisfied with the rehabilitative services provided [7]. In particular, they were most satisfied with the services catering to their physical well-being and functioning (such as medical services, occupational therapy, and physiotherapy); these services were also rated as highly important. On the other hand, while vocational counseling was ranked as one of the most important needs, it was also one of the least satisfactory services provided to them [7]. This, taken together with the economic, psychological, and cultural implications of returning to work discussed previously, suggests a critical need to improve rehabilitative efforts geared towards the reemployment of TBI patients. Certainly, more research and resources can be directed towards enabling TBI patients to recover their preinjury and maximize their postinjury occupational ability.

Additionally, given that the patients' main caregivers are their families, it is crucial to involve them in the rehabilitation process prior to the patient's hospital discharge. However, such family involvements remain somewhat low, and perhaps this explains their unpreparedness and lack of the know-how in caring for their brain-injured relatives $[52,53]$. The fact that TBI patients' disabilities may not be reflected in any atypical physical features (as discussed previously) makes this worse; these families may sometimes have unrealistic expectations of their brain-injured relatives upon discharge simply because they "look fine." As a result, tensions between the patient and his/her family members may arise, making it more difficult to care for the patient. Hence, to minimize such problems, prior to the patient's discharge, it would be helpful to inform and educate these families on the patient's impairments and the appropriate way to handle these impairments and care for the patient.

The few reported researches on interventions for braininjured populations conducted in Hong Kong have generally been effective in achieving certain specific goals such as the remediation of skills compromised by the injury and community integration. Despite the lack of local research on specifically rehabilitating TBI patients, these researches on other brain-injured populations do suggest that there are adequate resources, both in the community and in the health care infrastructure, to carry out similar rehabilitative efforts on TBI patients.

Certainly, future research on TBI rehabilitation in Hong Kong will be useful in informing and guiding the current practices and ultimately to better the lives of TBI patients. Intervention efforts utilizing cognitive-based rehabilitative approaches on brain-injured populations in Hong Kong have been scarce. In light of the possible impairments in various cognitive domains [18] and the fact that these impairments are a major concern among discharged braininjured patients in Hong Kong [53], there is huge potential for intervention work in this area. Additionally, with advances in neuroimaging, it will be useful for future interventions to track neuroplastic changes in the brain as a function of the intervention progress, so as to gain a better understanding of the brain mediators/mechanisms of such improvements in cognition, especially within the context of a neurological condition like TBI.

\section{Conclusions}

The present paper has reviewed the practice and research of TBI rehabilitation in Hong Kong. TBI rehabilitation in Hong Kong is carried out by a multidisciplinary team of health care professionals primarily within hospitals, as consistent with international guidelines. Despite limited resources, these rehabilitation services have generally satisfied most of the TBI patients' needs. However, there is room for improvement in rehabilitative efforts aimed at enabling them to return to work. While stigma is a major concern for these patients and their rehabilitation, it is hardly addressed in the current practices. Finally, research on TBI rehabilitation in Hong Kong is lacking; there is a need for more local research in this area to inform the current practice of TBI rehabilitation and more importantly to benefit TBI patients in Hong Kong.

\section{Conflict of Interests}

The authors declare that there is no conflict of interests regarding the publication of this paper.

\section{Acknowledgment}

This work is supported by funding from the KKHo International Charitable Foundation.

\section{References}

[1] A. I. R. Maas, N. Stocchetti, and R. Bullock, "Moderate and severe traumatic brain injury in adults," The Lancet Neurology, vol. 7, no. 8, pp. 728-741, 2008.

[2] R. C. Gardner and K. Yaffe, "Epidemiology of mild traumatic brain injury and neurodegenerative disease," Molecular and Cellular Neuroscience, vol. 66, pp. 75-80, 2015.

[3] C. Y. Kuo, T. H. Liou, K. H. Chang et al., "Functioning and disability analysis of patients with traumatic brain injury and spinal cord injury by using the World Health Organization disability assessment schedule 2.0," International Journal of Environmental Research and Public Health, vol. 12, no. 4, pp. 4116-4127, 2015.

[4] C. Konrad, A. J. Geburek, F. Rist et al., "Long-term cognitive and emotional consequences of mild traumatic brain injury," Psychological Medicine, vol. 41, no. 6, pp. 1197-1211, 2011.

[5] M. V. Morton and P. Wehman, "Psychosocial and emotional sequelae of individuals with traumatic brain injury: a literature review and recommendations," Brain Injury, vol. 9, no. 1, pp. 8192, 1995. 
[6] M. P. Dijkers, "Quality of life after traumatic brain injury: a review of research approaches and findings," Archives of Physical Medicine and Rehabilitation, vol. 85, supplement 2, pp. S21-S35, 2004.

[7] D. W. K. Man, E. W. T. Lee, E. C. H. Tong, S. C. S. Yip, W. F. Lui, and C. S. Lam, "Health services needs and quality of life assessment of individuals with brain injuries: a pilot crosssectional study," Brain Injury, vol. 18, no. 6, pp. 577-591, 2004.

[8] K. L. Leung and D. W. K. Man, "Prediction of vocational outcome of people with brain injury after rehabilitation: a discriminant analysis," Work, vol. 25, no. 4, pp. 333-340, 2005.

[9] A. Franulic, C. G. Carbonell, P. Pinto, and I. Sepulveda, "Psychosocial adjustment and employment outcome 2, 5 and 10 years after TBI," Brain Injury, vol. 18, no. 2, pp. 119-129, 2004.

[10] Census and Statistics Department, Hong Kong Annual Digest of Statistics 2011, Census and Statistics Department, Hong Kong, 2011.

[11] L.-W. Chu, I. Chi, and A. Y. Y. Chiu, "Incidence and predictors of falls in the Chinese elderly," Annals of the Academy of Medicine Singapore, vol. 34, no. 1, pp. 60-72, 2005.

[12] J. H. H. Yeung, A. A. Mikocka-Walus, P. A. Cameron et al., "Protection from traumatic brain injury in hormonally active women vs men of a similar age: a retrospective international study," Archives of Surgery, vol. 146, no. 4, pp. 436-442, 2011.

[13] S. R. Flanagan, M. R. Hibbard, and W. A. Gordon, "The impact of age on traumatic brain injury," Physical Medicine and Rehabilitation Clinics of North America, vol. 16, no. 1, pp. 163177, 2005.

[14] A. W. S. Leung, S. K. W. Cheng, A. K. Y. Mak, K.-K. Leung, L. S. W. Li, and T. M. C. Lee, "Functional gain in hemorrhagic stroke patients is predicted by functional level and cognitive abilities measured at hospital admission," NeuroRehabilitation, vol. 27, no. 4, pp. 351-358, 2010.

[15] J. H. H. Yeung, A. L. M. Chang, W. Ho et al., "High risk trauma in older adults in Hong Kong: a multicentre study," Injury, vol. 39, no. 9, pp. 1034-1041, 2008.

[16] H. M. Bramlett and W. D. Dietrich, "Long-term consequences of traumatic brain injury: current status of potential mechanisms of injury and neurological outcomes," Journal of Neurotrauma, 2014.

[17] L. Miller, Traumatic Brain Injury and Aggression, vol. 21, Elsevier, New York, NY, USA, 1st edition, 1994.

[18] G. Spitz, J. L. Ponsford, D. Rudzki, and J. J. Maller, "Association between cognitive performance and functional outcome following traumatic brain injury: a longitudinal multilevel examination," Neuropsychology, vol. 26, no. 5, pp. 604-612, 2012.

[19] F. Y. Kwok, T. M. C. Lee, C. H. S. Leung, and W. S. Poon, "Changes of cognitive functioning following mild traumatic brain injury over a 3-month period," Brain Injury, vol. 22, no. 10, pp. 740-751, 2008.

[20] R. C. K. Chan, R. Hoosain, T. M. C. Lee, Y. W. Fan, and D. Fong, "Are there sub-types of attentional deficits in patients with persisting post-concussive symptoms? A cluster analytical study," Brain Injury, vol. 17, no. 2, pp. 131-148, 2003.

[21] C. Shields, T. Ownsworth, A. O’Donovan, and J. Fleming, "A transdiagnostic investigation of emotional distress after traumatic brain injury," Neuropsychological Rehabilitation, 2015.

[22] G. W. Gregório, R. W. H. M. Ponds, S. M. J. Smeets et al., "Associations between executive functioning, coping, and psychosocial functioning after acquired brain injury," British Journal of Clinical Psychology, vol. 54, no. 3, pp. 291-306, 2015.
[23] N. Andelic, J. C. Arango-Lasprilla, P. B. Perrin et al., "Modeling of community integration trajectories in the first five years after traumatic brain injury," Journal of Neurotrauma, 2015.

[24] J. Lu, K. W. Gary, J. P. Neimeier, J. Ward, and K. L. Lapane, "Randomized controlled trials in adult traumatic brain injury," Brain Injury, vol. 26, no. 13-14, pp. 1523-1548, 2012.

[25] S. A. Reid-Arndt, R. G. Frank, and K. J. Hagglund, "Brain injury and health policy: twenty-five years of progress," Journal of Head Trauma Rehabilitation, vol. 25, no. 2, pp. 137-144, 2010.

[26] G. Simpson, R. Mohr, and A. Redman, "Cultural variations in the understanding of traumatic brain injury and brain injury rehabilitation," Brain Injury, vol. 14, no. 2, pp. 125-140, 2000.

[27] Legislative Council, "Resources allocation among hospital clusters by the Hospital Authority," Tech. Rep. CB(2)671/13-14(05), Legislative Council, 2014.

[28] G. K. K. Leung, "Trauma system in Hong Kong," Surgical Practice, vol. 14, no. 2, pp. 38-43, 2010.

[29] C. Y. Wong, C. T. Lui, F. L. So, K. L. Tsui, and S. Y. H. Tang, "Prevalence and predictors of under-diversion in the primary trauma diversion system in Hong Kong," Hong Kong Journal of Emergency Medicine, vol. 20, no. 5, pp. 276-286, 2013.

[30] N. K. Cheung, J. H. H. Yeung, J. T. S. Chan, P. A. Cameron, C. A. Graham, and T. H. Rainer, "Primary trauma diversion: initial experience in Hong Kong," Journal of Trauma-Injury, Infection and Critical Care, vol. 61, no. 4, pp. 954-960, 2006.

[31] W. K. Yuen and C. H. Chung, "Trauma care in Hong Kong," Trauma Quarterly, vol. 14, no. 3, pp. 241-247, 1999.

[32] K. W. Suen, A. M. L. Chang, and C. K. H. Au, "A regional study on primary trauma diversion in Hong Kong," Hong Kong Journal of Emergency Medicine, vol. 18, no. 2, pp. 92-100, 2011.

[33] T. H. Rainer, N. K. Cheung, J. H. H. Yeung, and C. A. Graham, "Do trauma teams make a difference? A single centre registry study," Resuscitation, vol. 73, no. 3, pp. 374-381, 2007.

[34] Hong Kong West Cluster Hospital Authority, "MacLehose Medical Rehabilitation Centre," 2007, http://www3.ha.org.hk/ mmrc/home.htm.

[35] H. M. Bramlett and W. D. Dietrich, "Pathophysiology of cerebral ischemia and brain trauma: similarities and differences," Journal of Cerebral Blood Flow and Metabolism, vol. 24, no. 2, pp. 133-150, 2004.

[36] Y. Rassovsky, Y. Levi, E. Agranov, M. Sela-Kaufman, A. Sverdlik, and E. Vakil, "Predicting long-term outcome following traumatic brain injury (TBI)," Journal of Clinical and Experimental Neuropsychology, vol. 37, no. 4, pp. 354-366, 2015.

[37] L. Mercier, T. Audet, R. Hébert, A. Rochette, and M.-F. Dubois, "Impact of motor, cognitive, and perceptual disorders on ability to perform activities of daily living after stroke," Stroke, vol. 32, no. 11, pp. 2602-2608, 2001.

[38] K. P. Liu, C. C. Chan, T. M. Lee, and C. W. Hui-Chan, "Mental imagery for promoting relearning for people after stroke: a randomized controlled trial," Archives of Physical Medicine and Rehabilitation, vol. 85, no. 9, pp. 1403-1408, 2004.

[39] D. Y. L. Chan, C. C. H. Chan, and D. K. S. Au, "Motor relearning programme for stroke patients: a randomized controlled trial," Clinical Rehabilitation, vol. 20, no. 3, pp. 191-200, 2006.

[40] P. A. Logan, J. R. F. Gladman, and K. A. Radford, "The use of transport by stroke patients," British Journal of Occupational Therapy, vol. 64, no. 5, pp. 261-264, 2001.

[41] F. K. Y. Wong and S. M. Yeung, "Effects of a 4-week transitional care programme for discharged stroke survivors in Hong Kong: a randomised controlled trial," Health and Social Care in the Community, 2014. 
[42] J. C. K. Lai, J. Woo, E. Hui, and W. M. Chan, "Telerehabilitation- a new model for community-based stroke rehabilitation," Journal of Telemedicine and Telecare, vol. 10, no. 4, pp. 199-205, 2004.

[43] P.-H. Chau, F. Yeung, T.-W. Chan, and J. Woo, "A quasiexperimental study on a new service option for short-term residential care of older stroke patients," Clinical Interventions in Aging, vol. 8, pp. 1167-1175, 2013.

[44] S.-F. Tam and W.-K. Man, "Evaluating computer-assisted memory retraining programmes for people with post-head injury amnesia," Brain Injury, vol. 18, no. 5, pp. 461-470, 2004.

[45] R. C. K. Chan, R. Hoosain, and T. M. C. Lee, "Talking while performing a task: a better attentional performance in patients with closed head injury?" Journal of Clinical and Experimental Neuropsychology, vol. 24, no. 5, pp. 695-704, 2002.

[46] K. D. Cicerone, D. M. Langenbahn, C. Braden et al., "Evidencebased cognitive rehabilitation: updated review of the literature from 2003 through 2008," Archives of Physical Medicine and Rehabilitation, vol. 92, no. 4, pp. 519-530, 2011.

[47] M. A. Linden, R. J. Rauch, and I. R. Crothers, "Public attitudes towards survivors of brain injury," Brain Injury, vol. 19, no. 12, pp. 1011-1017, 2005.

[48] S. Tam, A. McKay, S. Sloan, and J. Ponsford, "The experience of challenging behaviours following severe TBI: a family perspective," Brain Injury, vol. 29, no. 7-8, pp. 813-821, 2015.

[49] A. Jacoby, W. Wang, T. D. Vu et al., "Meanings of epilepsy in its sociocultural context and implications for stigma: findings from ethnographic studies in local communities in China and Vietnam," Epilepsy and Behavior, vol. 12, no. 2, pp. 286-297, 2008.

[50] J. R. Fann, B. Burington, A. Leonetti, K. Jaffe, W. J. Katon, and R. S. Thompson, "Psychiatric illness following traumatic brain injury in an adult health maintenance organization population," Archives of General Psychiatry, vol. 61, no. 1, pp. 53-61, 2004.

[51] J. Hsu, "Family therapy for the Chinese: problems and strategies," in Chinese Societies and Mental Health, T. Y. Lin, W. S. Tseng, and E. L. Yeh, Eds., Oxford University Press, Hong Kong, 1995.

[52] D. W. K. Man, "Family caregivers' reactions and coping for persons with brain injury," Brain Injury, vol. 16, no. 12, pp. 10251037, 2002.

[53] H. M. K. Tam and T. M. C. Lee, "A post-discharge service for brain injury patients in Hong Kong," Chinese Journal of Rehabilitation Medicine, vol. 26, no. 12, pp. 1098-1103, 2011.

[54] K. F. Chung and M. C. Wong, "Experience of stigma among Chinese mental health patients in Hong Kong," Psychiatric Bulletin, vol. 28, no. 12, pp. 451-454, 2004.

[55] Social Welfare Department, Social Security Allowance (SSA) Scheme, Social Welfare Department, 2012, http://www.swd.gov .hk/doc/social-sec/SSAP0612e.pdf.

[56] J. McClure, "The role of causal attributions in public misconceptions about brain injury," Rehabilitation Psychology, vol. 56, no. 2, pp. 85-93, 2011.

[57] D. W. K. Man, A. S. F. Tam, and E. P. Y. Li, "Exploring selfconcepts of persons with brain injury," Brain Injury, vol. 17, no. 9, pp. 775-788, 2003.

[58] L. G. Irwin and D. G. Fortune, "Schools-based interventions for reducing stigmatization of acquired brain injury: the role of interpersonal contact and visible impairment," Archives of Clinical Neuropsychology, vol. 29, no. 2, pp. 194-205, 2014.
[59] Royal College of Physicians and British and Society of Rehabilitation Medicine, Rehabilitation Following Acquired Brain Injury National Clinical Guidelines, RCP, BSRM, London, UK, 2003. 


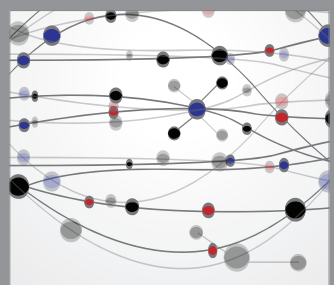

The Scientific World Journal
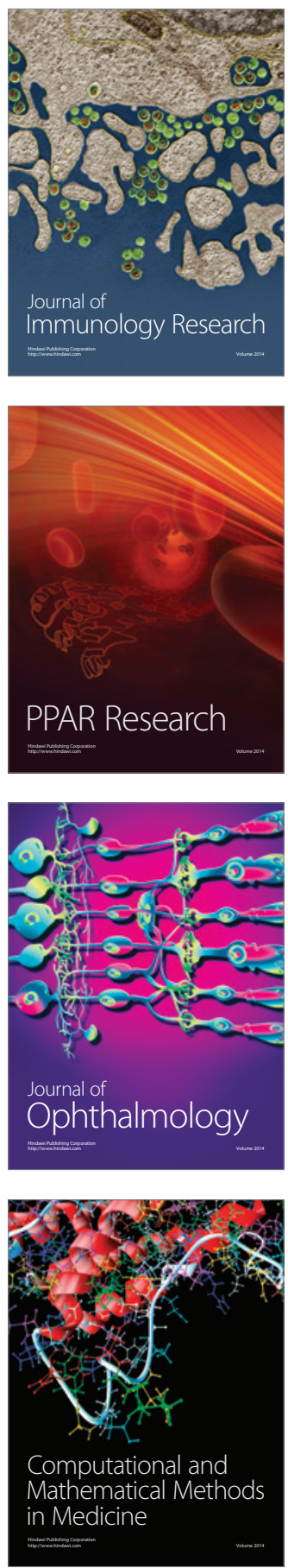

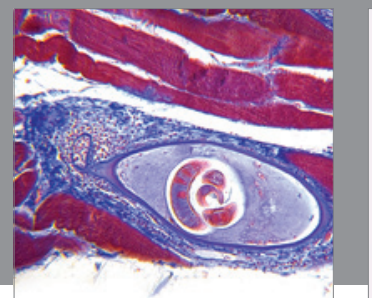

Gastroenterology

Research and Practice
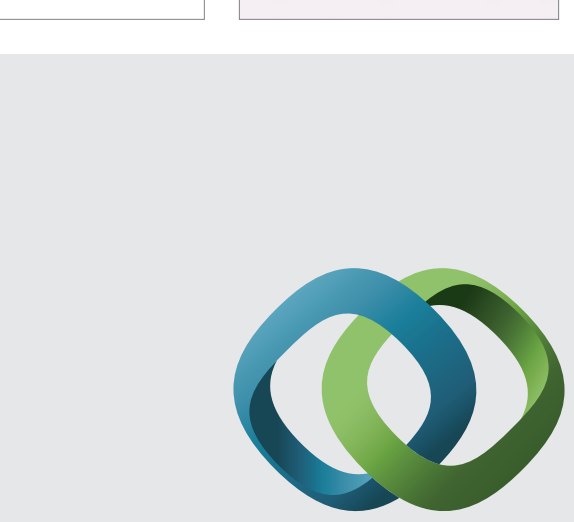

\section{Hindawi}

Submit your manuscripts at

http://www.hindawi.com
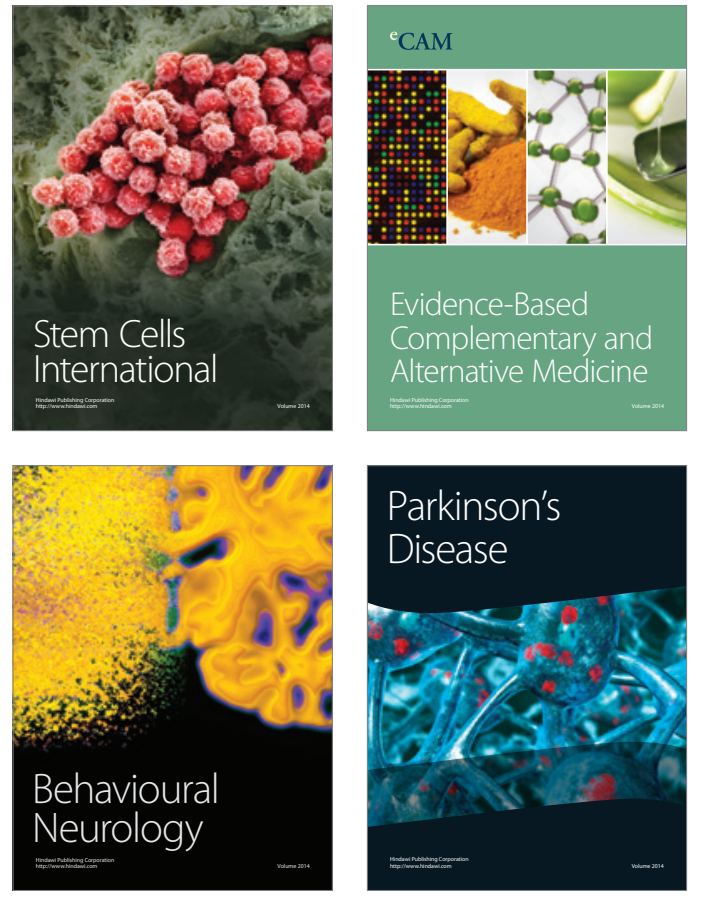
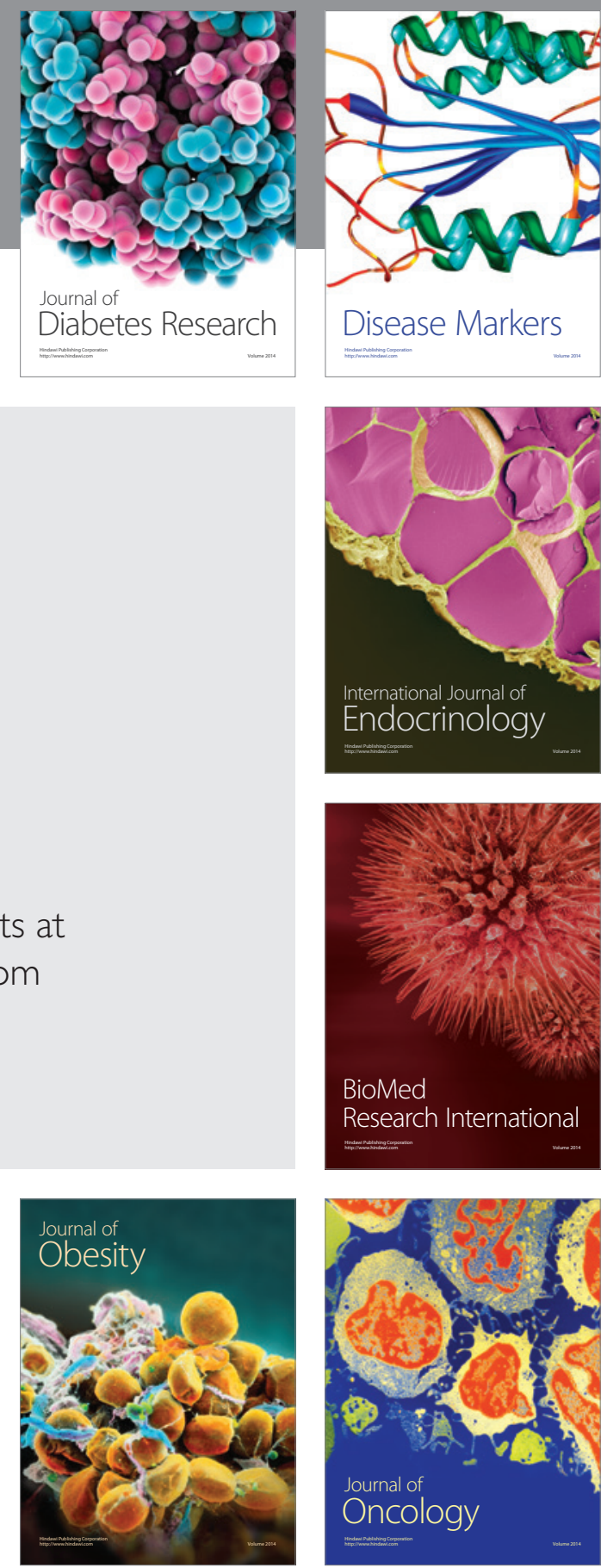

Disease Markers
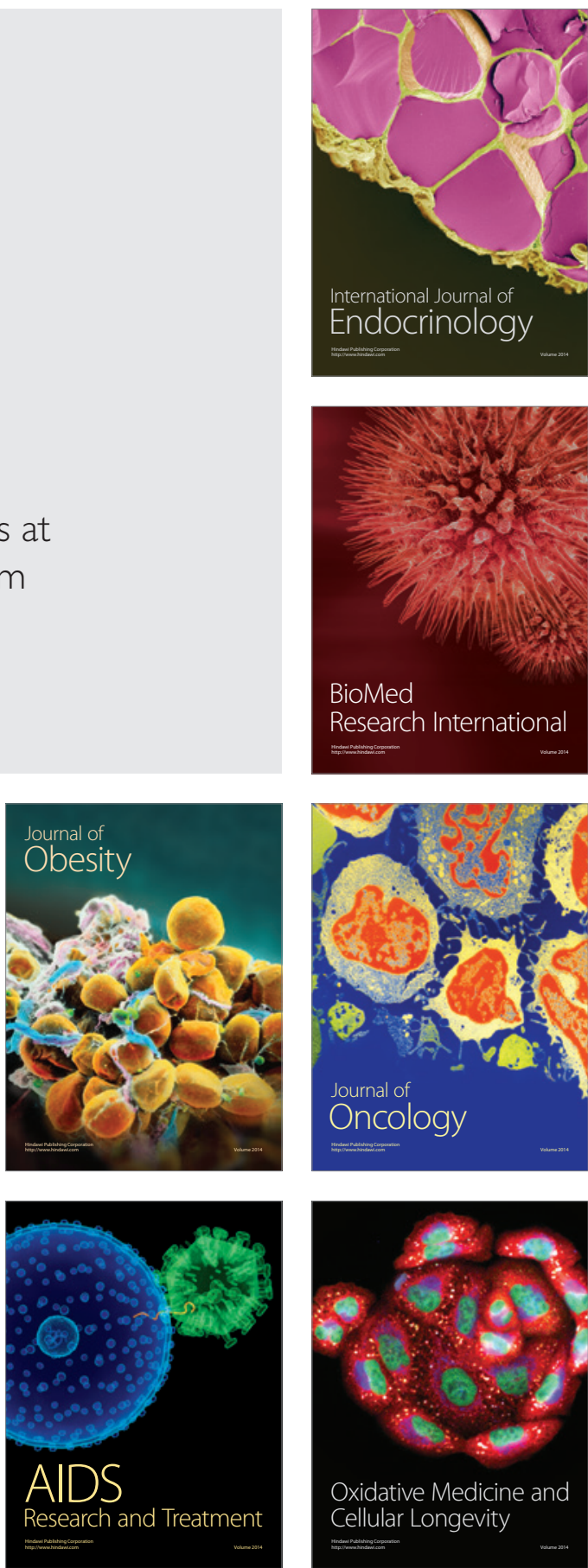